\title{
The aromatic universe
}

Thirty-four years ago, examining the chemical nature of a sooty residue made by vaporizing carbon in an inert helium atmosphere, the chemist Harold Kroto and colleagues discovered molecules of pure carbon made of 60 or 70 atoms bound together into an approximately spherical shape. These became known as fullerenes, or 'buckyballs' - and they set the stage for the discovery of a host of other carbon forms, including carbon nanotubes and graphene. Before Kroto's work, the known allotropes of carbon were limited to graphite, diamond and various amorphous forms.

Back then, one might have suspected that these beautiful fullerenes reflected the triumph of the human imagination, improving on nature's own creations. Not Kroto. He expected that nature would have surely discovered them first, and he was right. As the technology for astrophysical infrared spectroscopy improved, NASA's Spitzer infrared telescope first detected signatures of $\mathrm{C}_{60}$ and $\mathrm{C}_{70}$ in 2010 in a cloud of cosmic dust surrounding a star 6,500 light years away. Since then, they've been found essentially everywhere in the dust and gas of the interstellar medium (ISM), near our own Galaxy and others far away.

These observations have kicked off an effort to understand how this sparse and cold medium could have given rise not only to these complex molecules, but also to a host of large molecules made primarily of carbon, as well as to clarify the role they may play in the eventual formation of stars and the evolution of galaxies. As astrochemists Alessandra Candian, Junfeng Zhen and Xander Tielens describe in a recent review (Phys. Today 71, 38; 2018), full understanding of this "aromatic universe" is still missing. But researchers have far more settled ideas, and expect a leap forward as vast new data at higher resolution begins to come in from NASA's James Webb Space Telescope, due to be launched in 2021.

The fullerenes are closely related to a class of molecules that chemists call polycyclic aromatic hydrocarbons (PAHs) fused benzene rings joined together into flat sheets, with hydrogen atoms attached to their edges. On Earth, PAHs arise naturally in the burning of organic matter, such as coal and tar, and end up in the blackened crust of many cooked foods. With the inclusion of other atoms such as nitrogen or oxygen in small numbers, these PAHs form thousands of key molecular building blocks.
Outside Earth, molecules of this class turn up everywhere in the ISM, which seems to be a fairly sooty place. If illuminated by ultraviolet (UV) radiation, these molecules absorb photons, move into higher-energy states, and then relax again through the emission of lower-energy infrared (IR) photons associated with vibrational motions. The resulting mid-IR spectra show a series of strong emission features, generally known as the aromatic infrared bands (AIBs). Their main features reflect the stretching of $\mathrm{C}-\mathrm{H}$ and $\mathrm{C}-\mathrm{C}$ bonds, as well as in-plane and outof-plane $\mathrm{C}-\mathrm{H}$ bending motions.

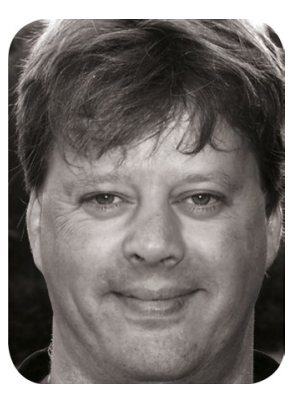

The discovery of fullerenes, or 'buckyballs', set the stage for the discovery of a host of other carbon forms, including carbon nanotubes and graphene.

Such spectra have been observed in a wide variety of settings, including the dusty disks around young stars, the general ISMs of galaxies, and also in very distant regions reflecting the Universe as it was 3.3 billion years ago. Before 2010, however, the largest molecules detected in the ISM held only 12 atoms. In 2010, astronomer Jan Cami at the University of Western Ontario and his colleagues took an IR spectrum of the young planetary nebula $\mathrm{Tc} 1$, finding strong vibrational transitions at 7.0, 8.6, 17.4 and $18.9 \mu \mathrm{m}$, peaks that they recognized as a close match for $\mathrm{C}_{60}$ and $\mathrm{C}_{70}$. They estimated that these chemical species made up a few per cent of the carbon in this region.

Ionized states of $\mathrm{C}_{60}$ also lie behind other spectral signatures. So-called diffuse interstellar bands (DIBs) are a family of around 500 absorption features from the near-UV to the near-IR seen in the spectra of interstellar clouds in the Milky Way and other galaxies. The origin of most of the DIBs remains unknown, but researchers have long believed they arise from electronic transitions in molecules. Earlier this year, spectral signatures of ionized $\mathrm{C}_{60}$ molecules were detected with the Hubble Space Telescope.

If the fullerenes are so common, how were they formed in the ISM? The most obvious possibility, as Candian and colleagues note, is through some sequence of gas-phase, bottom-up reactions, as larger molecules assemble from smaller molecules. But this picture seems difficult to sustain for a variety of reasons. For example, chemists have found it difficult to find pathways to large molecules that are plausible in the physical environment of the ISM. One organic molecule, vinylacetylene, which contains four carbon and four hydrogen atoms, could be a key additive component able to build larger molecules. But this seems very rare in the relevant environments. Logically, astrochemists believe, it seems difficult to build PAH molecules containing 50 carbon atoms or more in a bottom-up fashion.

An alternative view suggests that large PAHs and fullerenes in space form in some very different downstream process, rather than being built up from smaller parts. One idea is they are produced in the material ejected from so-called asymptotic giant branch stars, within which densities and temperatures lead to chemistry quite similar to that in a combustion engine. Those stars eject much of their carbon into space, mostly in the form of soot. This pathway suggests that interstellar PAHs would be molecular intermediaries or by-products of the soot formation process. Stellar winds would then transport these molecular species into the general ISM.

Research in this field will get a huge boost when the James Webb Space Telescope goes up in 2021, as it will be equipped with instruments designed to study the aromatic universe with high spectral and spatial resolution. It should also make it possible to measure the star formation rate in dusty, highly obscured regions of IR galaxies. Astrochemists also believe that PAHs are abundant in protoplanetary disks, and the limited data currently available suggest that they change chemically while being transported from the general ISM to those protoplanetary disks. With the new telescope, IR signatures should reveal the molecular structure of the PAH family on route to planetary formation.

In the end, we may find a surprise: for all the exotic astrophysics driving the Universe, much of the basic chemistry of planetary formation may involve variations of the same smoky and sooty combustion of organic matter we know so well on Earth.

\section{Mark Buchanan}

Published online: 1 October 2019

https://doi.org/10.1038/s41567-019-0681-7 